\title{
Preventing Assembly and Crystallization of Alkane Acids at Silica- Bitumen Interface to Enhance Interfacial Resistance to Moisture Damage
}

Albert M. Hung, ${ }^{\text {a }}$ Farideh Pahlavan ${ }^{\mathrm{a}}$, Sheyda Shakiba, ${ }^{\mathrm{b}}$ Shery L. Y. Chang ${ }^{\mathrm{a}}$, Stacey M. Louie, ${ }^{\mathrm{b}}$ Elham H. Fini ${ }^{\mathrm{a}}{ }^{*}$

a. Arizona State University, Tempe, AZ 85287-3005, Phone: 480-965-4273, USA

b. University of Houston, 4726 Calhoun Rd., Houston, TX 77204-4003, USA

* Corresponding author: E. H. Fini (efini@asu.edu)

Number of Figures: 7

Number of Tables: 1

Number of Pages: 6
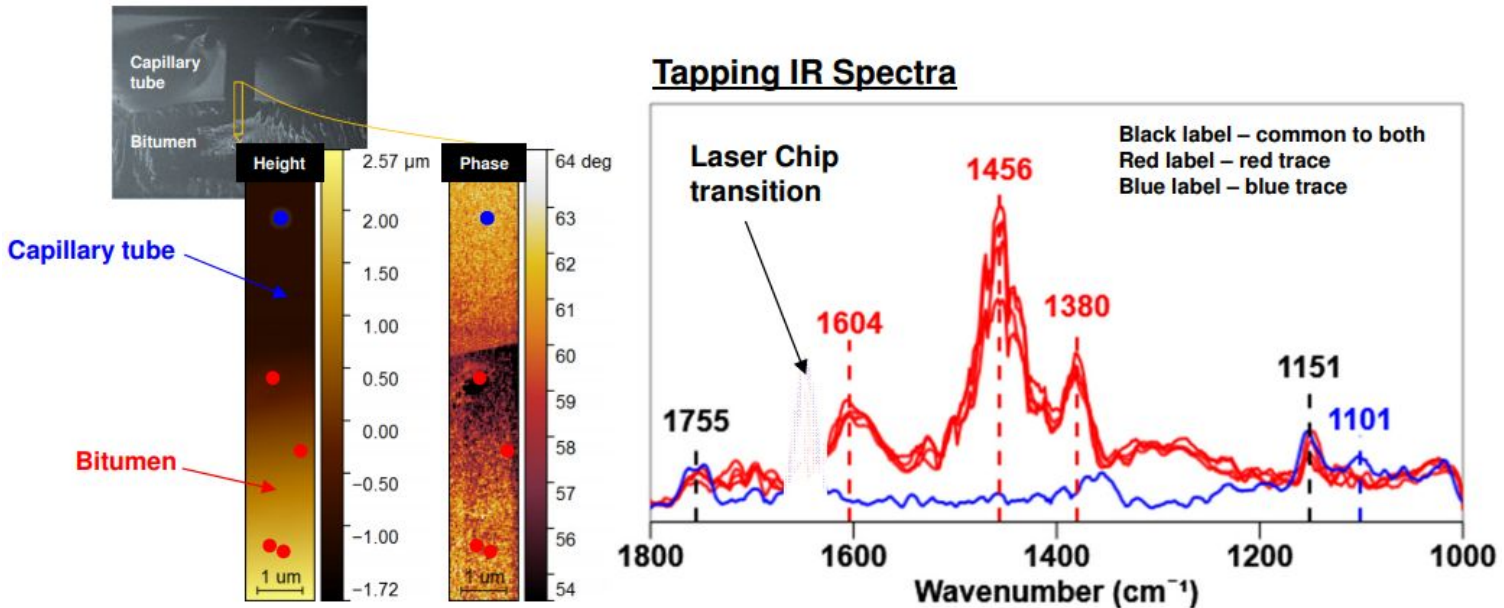

Figure S1. Tapping AFM-IR spectra of HDA/Bio-binder-doped bitumen at the glass interface. Spectra taken on the bitumen area are presented in red. A spectrum taken on the glass is presented in blue. (Some absorption at $1755 \mathrm{~cm}^{-1}$ may be due to contamination on the tip or sample surface.) 


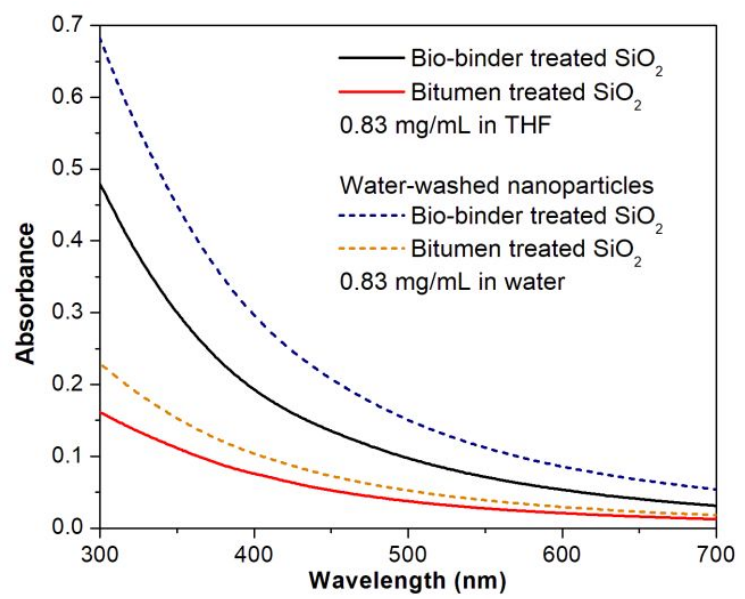

Figure S2. UV-visible absorption spectra of $\mathrm{SiO}_{2} \mathrm{NPs}$ treated with Bio-binder or bitumen, before (solid lines) and after (dashed lines) rinsing with deionized (DI) water. To rinse the NPs, 3-8 mg of NPs are dispersed by 5-10 min ultrasonication in $1 \mathrm{~mL}$ of DI water, centrifuged down, and separated from the supernatant (which is discarded). This cycle is repeated two more times before the NPs are resuspended in $1 \mathrm{~mL}$ of DI water. For both samples of treated particles, the absorbance intensity appears to increase after water washing. This perceived increase is due to a solvochromic red-shift of the absorbance spectra in water compared to THF. The data suggest that the polyaromatic adsorbates in both cases are not easily washed off of silica substrates by water alone.

(a)<smiles>CNC(Cc1ccc(O)c(O)c1)C(=O)O</smiles>

(b)<smiles>CNC(CCCCN)C(=O)O</smiles>

Figure S3. Chemical structures of (a) 3,4-dihydroxyphenylalanine and (b) lysine as peptide residues. 


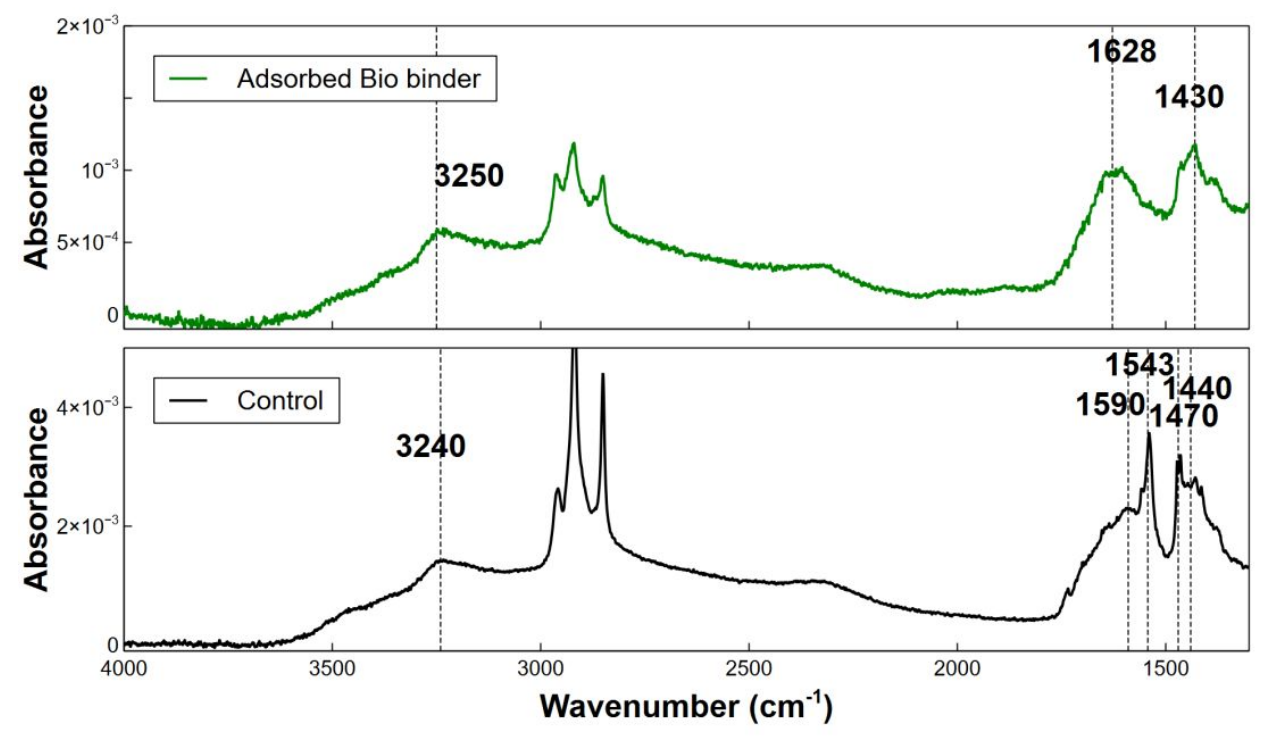

Figure S4. ATR-FTIR spectra of dried $\mathrm{SiO}_{2}$ NPs with adsorbed Bio-binder (top), and a control of dried, poorly soluble Bio-binder residue. Both samples were prepared with a solution of $1 \%$ Biobinder in THF, and solid NPs or Bio-binder residue was washed five times with THF, followed by deposition and drying to the ATR substrate. The clean, dry ZnSe ATR substrate was background-subtracted from the spectra.

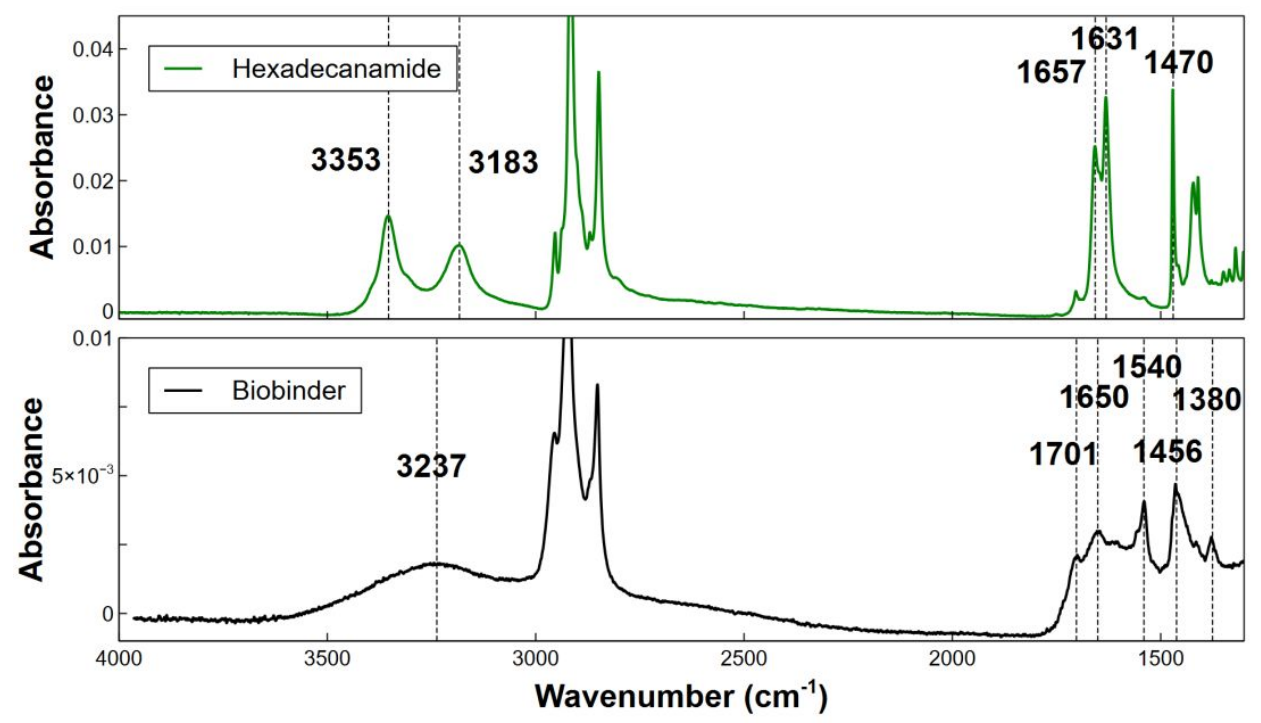

Figure S5. ATR-FTIR spectra of dry hexadecanamide and Bio-binder, with the spectrum of the ATR crystal subtracted as the background. In the hexadecanamide (top), the peaks at (1470, 1631, and 1657) $\mathrm{cm}^{-1}$ correspond to $\mathrm{C}-\mathrm{N}$ stretching, $\mathrm{N}-\mathrm{H}$ bending, $\mathrm{C}=\mathrm{O}$ stretching, and the peaks around (3183 and 3353) $\mathrm{cm}^{-1}$ correspond to $\mathrm{N}-\mathrm{H}$ stretching. In the Bio-binder (bottom), the broad peak around (3300 to 3000 ) $\mathrm{cm}^{-1}$ may be due to different functional groups such as aromatics, alcohols, and amines, and the region from (1800 to 1600) $\mathrm{cm}^{-1}$ can be attributed to organic molecules containing nitrogen or oxygen like carbonyl, amine, and aldehyde groups. 


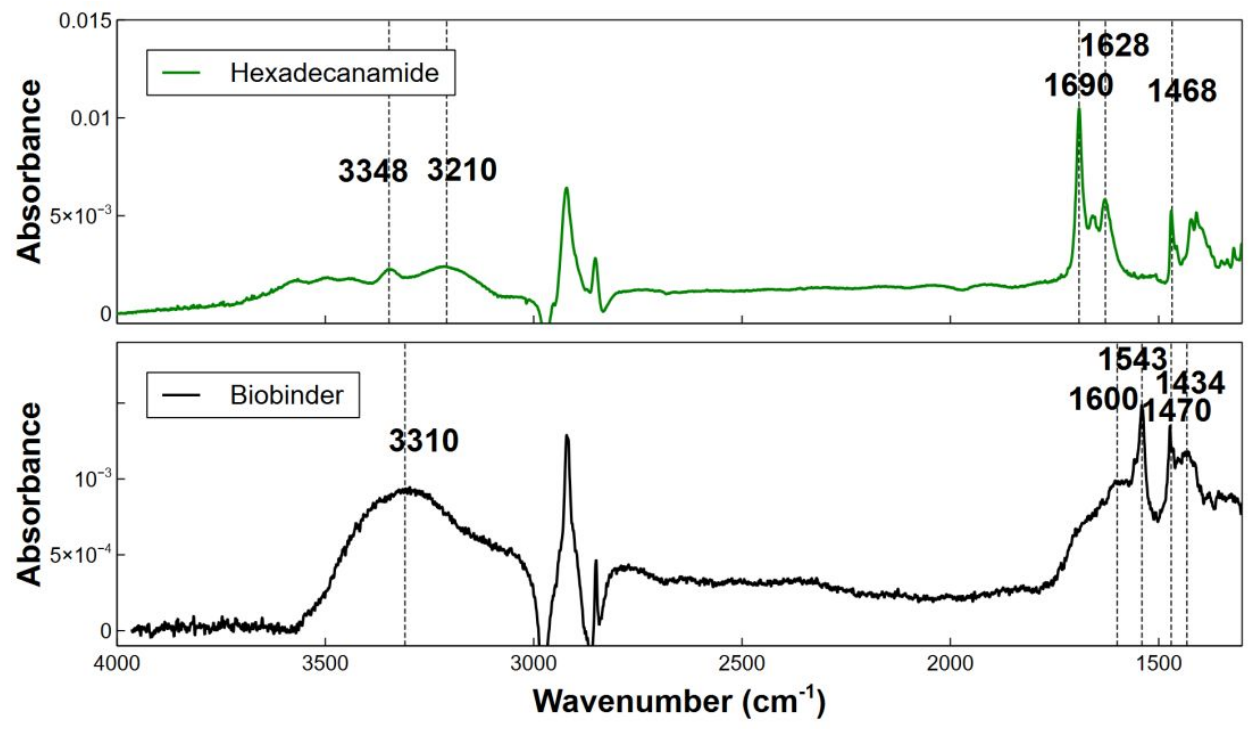

Figure S6. ATR-FTIR spectra of hexadecanamide and Bio-binder in THF. After collecting the spectra, the spectrum of pure liquid THF was subtracted from them. The peak assignments are as denoted in Figure S5 for the dried compounds, but shifts in peak locations and relative peak intensities are observed due to interactions with THF. Hence, adsorption results were compared to the spectra of the reference compound or bulk Bio-binder in THF, as shown here.
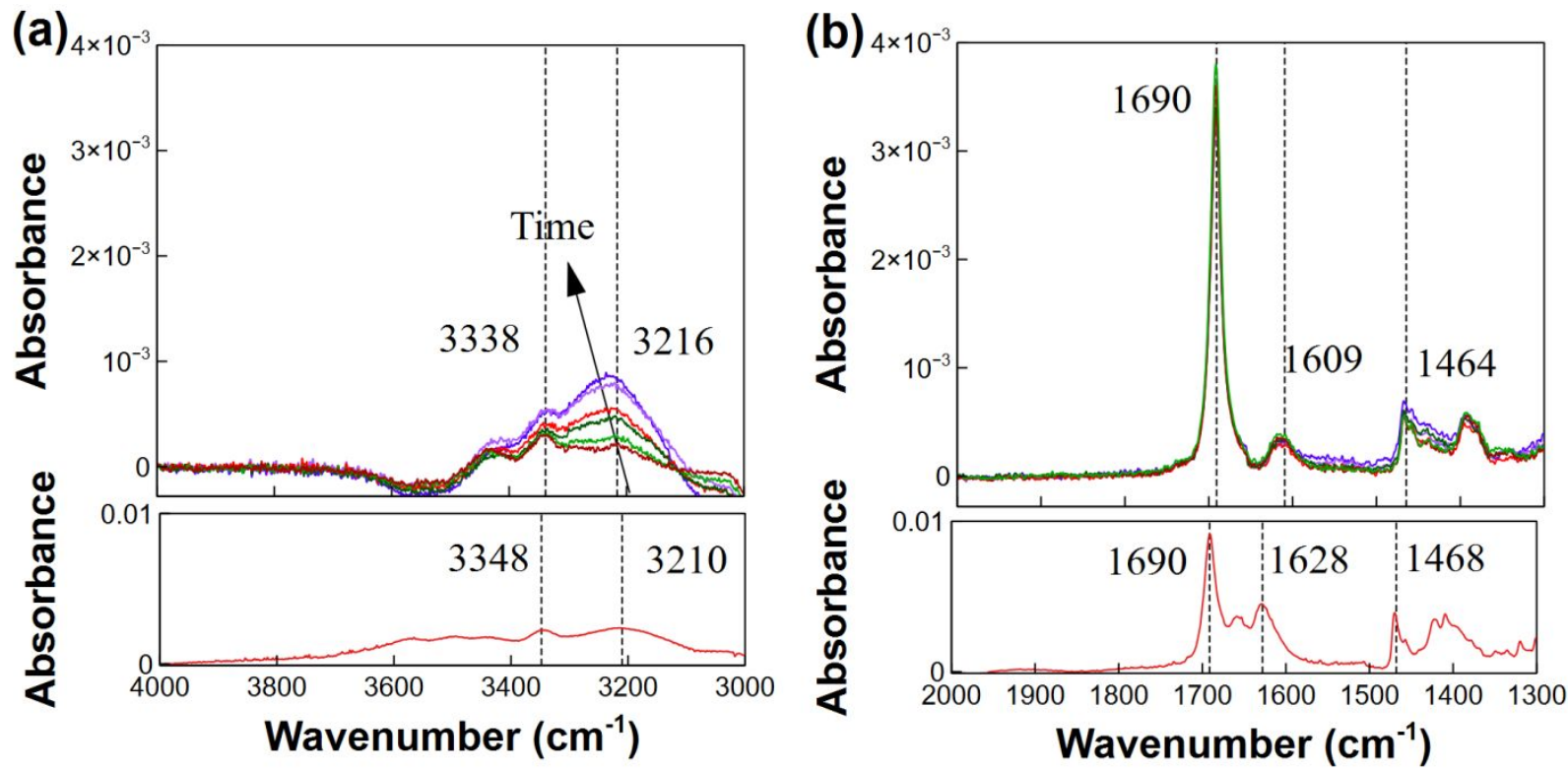

Figure S7. In situ ATR-FTIR experiment for adsorption of hexadecanamide (1\%) onto $\mathrm{SiO}_{2} \mathrm{NPs}$ from THF. The spectra were collected every 10 minutes for 110 minutes (spectra for every 20 minutes are presented here). To investigate the kinetics of adsorption, two regions from (a) (4000 to 3000$) \mathrm{cm}^{-1}$ and (b) (2000 to 1300) $\mathrm{cm}^{-1}$ were plotted. The spectrum of liquid THF over the NPs 
was subtracted from data. The bottom figures show the spectrum of dissolved hexadecanamide in THF, after subtracting the spectrum of pure liquid THF. Changes in relative peak heights as well as shifts in the peak locations of the $\mathrm{N}-\mathrm{H}$ stretching modes from (3348 and 3210) $\mathrm{cm}^{-1}$ in THF to (3338 and 3216) $\mathrm{cm}^{-1}$ after adsorption, and the $\mathrm{N}-\mathrm{H}$ bending mode from $1628 \mathrm{~cm}^{-1}$ in THF to $1609 \mathrm{~cm}^{-1}$ after adsorption, suggest strong interaction of these bonds with the $\mathrm{SiO}_{2} \mathrm{NPs}$. All spectra were vertically aligned at $4000 \mathrm{~cm}^{-1}$ in (a) and $2000 \mathrm{~cm}^{-1}$ in (b) but otherwise are presented at a common scale. 
Table S1. Molecular configurations and adsorption energies $\left(\mathrm{E}_{\mathrm{ads}}, \mathrm{kcal} / \mathrm{mol}\right)$ of selected molecular adsorbates on a hydroxylated quartz (001) surface. The simplified Bio-binder compounds in this table are named T-BioB (truncated Bio-binder).
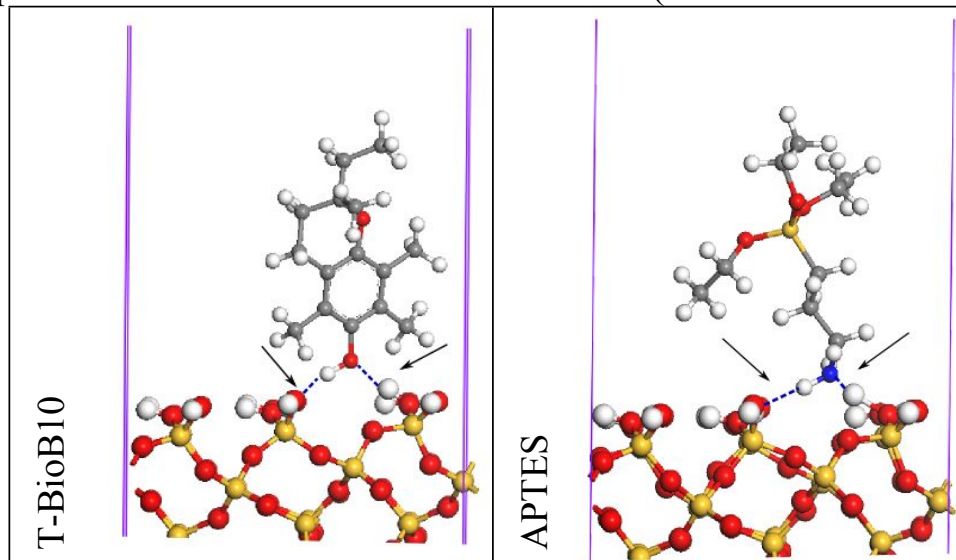

$E_{\text {ads }}=-51.9$

$E_{\text {ads }}=-36.3$
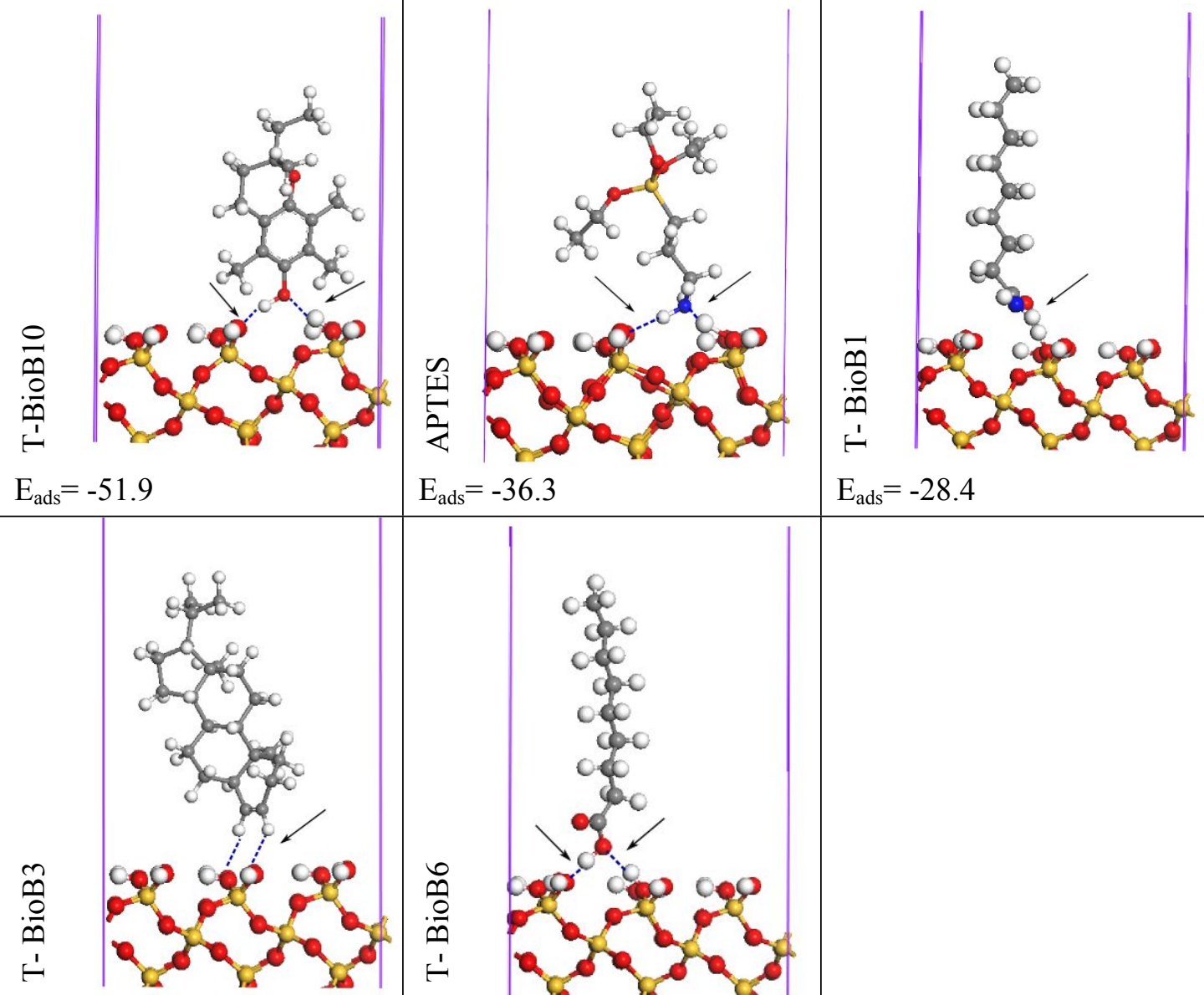

$E_{\text {ads }}=-27.5$

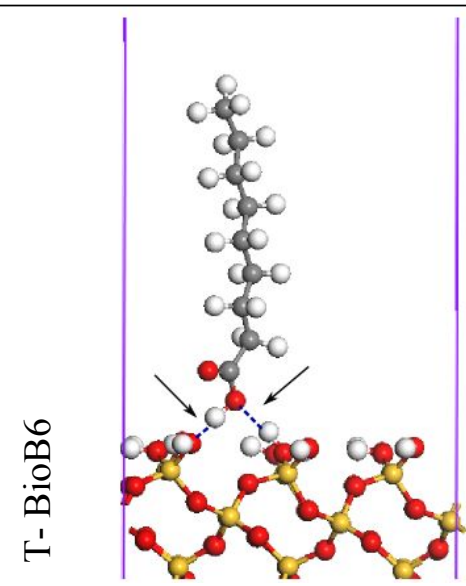

$\mathrm{E}_{\mathrm{ads}}=-28.4$

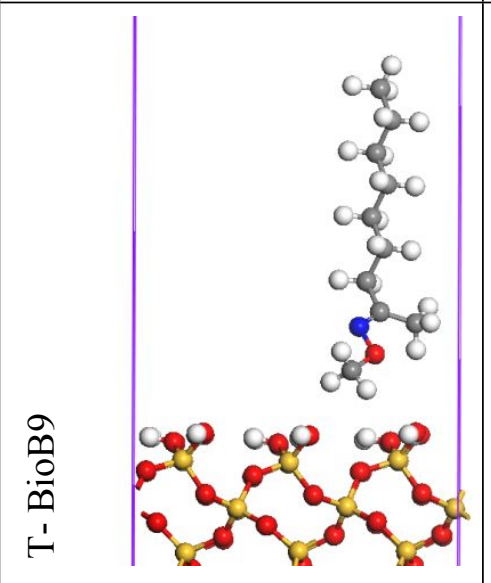

$\mathrm{E}_{\mathrm{ads}}=-20.8$

$E_{\text {ads }}=-21.3$

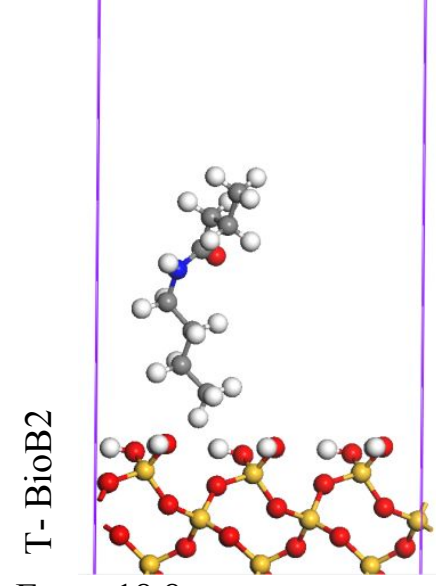

$\mathrm{E}_{\mathrm{ads}}=-18.8$

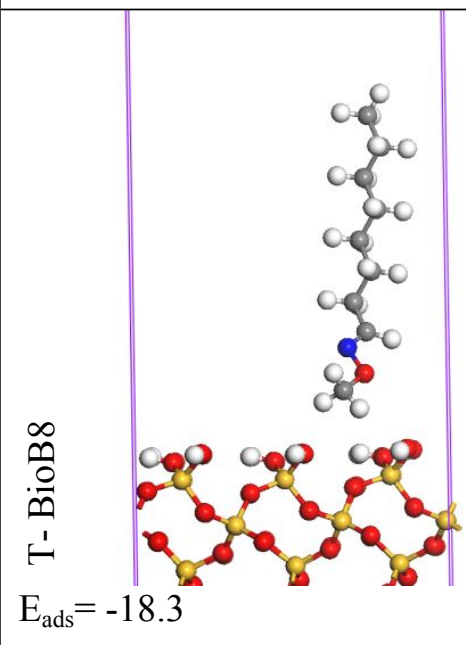

